\title{
Associations between childhood trauma, bullying and psychotic symptoms among a school-based adolescent sample
}

Ian Kelleher, Michelle Harley, Fionnuala Lynch, Louise Arseneault, Carol Fitzpatrick and Mary Cannon

\section{Background}

Children and adolescents who report psychotic symptoms appear to be at increased risk for psychotic disorders in adulthood - a putative 'symptomatic' high-risk group. However, little research has investigated whether those in this high-risk population have increased rates of exposure to traumatic events in childhood, as seen in patients who have a psychotic illness.

\section{Aims}

To examine whether adolescents with psychotic symptoms have an increased rate of traumatic experiences.

\section{Method}

Psychiatric interviews were carried out with 211 adolescents aged between 12 and 15 years and their parents as part of a population-based study. The interview enquired about a number of early traumatic events including physical and sexual abuse, exposure to domestic violence and bullying.

\section{Results}

Fourteen adolescents $(6.6 \%$ of those interviewed) reported experiencing at least one psychotic symptom. Adolescents who reported psychotic symptoms were significantly more likely to have been physically abused in childhood, to have been exposed to domestic violence and to be identified as a bully/victim (that is, both a perpetrator and victim of bullying) than those who did not report such symptoms. These findings were not confounded by comorbid psychiatric illness or family history of psychiatric history.

\section{Conclusions}

Our findings suggest that childhood trauma may increase the risk of psychotic experiences. The characteristics of bully/ victims deserve further study.

\section{Declaration of interest}

None. Funding detailed in Acknowledgements.
A proposed causal association between childhood trauma and later psychosis has raised much debate within the psychiatric literature over recent years. ${ }^{1-6}$ With some notable exceptions, ${ }^{5,6}$ the literature to date has been open to the risk of recall bias as most studies were based on treatment samples and used retrospective assessment of trauma. Although a number of studies indicate that individuals with psychotic disorders are no less likely to be accurate in recalling abusive experiences than the general population, ${ }^{7,8}$ some researchers still voice reliability concerns. ${ }^{2}$ One solution to this methodological problem is to examine the association between childhood trauma and psychosis in a population-based sample of people who have not yet had contact with the mental health services. The account of trauma is therefore unbiased by diagnosed outcome. We analysed data from a population-based sample of adolescents recruited from schools in Dublin, Ireland, to investigate whether there was an association between childhood traumatic experiences and the presence of psychotic symptoms. Research on long-term outcomes suggests there are increased rates of adult psychotic illness among adolescents reporting psychotic symptoms. ${ }^{9}$ Therefore, adolescents who report psychotic symptoms can be conceptualised as a 'high-risk' group for psychotic illness and this paradigm can be used to explore early risk factors for psychosis vulnerability. We wished to examine a range of traumatic experiences among such a cohort to investigate the relationship between psychotic symptoms in adolescence and childhood trauma.

\section{Method}

\section{Participants}

The Challenging Times study ${ }^{10,11}$ was established to investigate the prevalence of psychiatric disorders among Irish adolescents aged
12-15 years. The study was carried out in the geographical catchment area of a child and adolescent mental health team in North Dublin with a population of 137000 . After describing the study to participants, written informed consent was obtained from their parent or guardian. In brief, 743 pupils (52\% of the total school population in that area) were screened for psychiatric symptoms using the Strengths and Difficulties Questionnaire ${ }^{12,13}$ and the Children's Depression Inventory. ${ }^{14}$ Eight primary schools were selected using a stratified random sampling technique, stratified in order to approximate the socio-economic profile of the geographical area population. Overall, 140 adolescents scored above threshold on these instruments, indicating high risk of having mental health problems. Of these, 117 (84\%) agreed to attend a full psychiatric interview. A comparison group of 173 adolescents, matched for gender and school were also invited to attend, of whom 94 (54\%) agreed.

Ethical approval for the study was granted by the medical ethics committee of the Mater Misericordiae University Hospital, Dublin, Ireland. The study was supported by the multidisciplinary child and adolescent mental health team covering the geographical area. The protocol ensured that any child who was deemed to be in need of a clinical service could be referred to the team.

\section{Interview instrument}

The interview schedule used in this study was the Schedule for Affective Disorders and Schizophrenia for School-Aged Children, Present and Lifetime Versions (K-SADS). ${ }^{15}$ The K-SADS is a wellvalidated, semi-structured research diagnostic interview for the assessment of all Axis 1 psychiatric disorders in children and adolescents. Children and parents were interviewed separately, both answering the same questions. Interviews were conducted by two psychologists and one psychiatrist who were trained in 
the use of the K-SADS. Interrater reliability for the K-SADS was estimated as $>90 \%$ in this study. ${ }^{11}$

\section{Exposure measurement}

As part of the K-SADS interview the following childhood traumatic experiences were assessed. Interviews were conducted with parents and children separately.

\section{Childhood abuse}

Both child physical and sexual abuse were assessed as part of the $\mathrm{K}-\mathrm{SADS}$ interview. Children were asked the following questions in relation to physical abuse: 'When your parents got mad at you, did they hit you? Have you ever been hit so that you had bruises or marks on your body, or were hurt in some way? What happened?' They were asked the following questions in relation to sexual abuse: 'Did anyone ever touch you in your private parts when they shouldn't have? What happened? Has someone ever touched you in a way that made you feel bad?'.

Parents were asked the same questions appropriately modified. A disclosure of physical or sexual abuse from the parent was taken as evidence of a history of child abuse, regardless of whether it was also disclosed by the child. There were no cases where the parent disputed the occurrence of abuse that had been disclosed by the child.

\section{Domestic violence}

Exposure to domestic violence was assessed in the post-traumatic stress disorder section: 'Some kids' parents have a lot of nasty fights. They call each other bad names, throw things, and threaten to do bad things to each other. Did your parents ever get in really bad fights? Tell me about the worst fight you remember your parents having. What happened?'. Again, parents were asked the same questions, appropriately modified. A disclosure of physical violence between parents/step-parents from the parent was taken as evidence of a history of domestic violence. There were no cases where the parent disputed the occurrence of domestic violence that had been disclosed by the child.

\section{Bullying}

Bullying was assessed as part of the K-SADS Social Relations section; parents and children were specifically asked: 'Have you ever been bullied?' and 'Have you ever been accused of being a bully?. A positive response from either the parent or child was taken as evidence of a history of being a bully or being a victim of bullying. The 'victim of bullying' group included all those who responded positively to being bullied (regardless of whether they were also perpetrators of bullying). The 'perpetrator of bullying' group included all those who responded positively to being bullies (regardless of whether they were also victims of bullying).

\section{Outcome measures}

The Psychosis section of the K-SADS asks about the child's experience of hallucinations and delusions. Screen questions are detailed in the Appendix. Responses to these questions were recorded on the interview sheet. Three psychiatrists (M.H., F.L. and M.C.) and one psychologist (I.K.) examined these responses and concurred that the symptoms seemed genuine in content. All psychotic experiences were reported by the child. In no case did the parents report such symptoms in their child.

\section{Socio-economic status and family history of psychiatric illness}

Socio-economic status of each participant was determined using parental occupation assessed according to the Irish Social Class Scale. ${ }^{16}$ We divided the sample into two major groups according to social class: the first group contained socio-economic status groups 1 and 2 (professional/managerial) and the second group contained groups 3-7: (non-manual skilled, skilled manual, semi-skilled manual, unskilled manual and unemployed). The K-SADS interview includes a routine screening section for family history of psychiatric illness, which was used in the present study.

\section{Statistical analysis}

First, we used chi-squared tests to compare the socio-demographic and clinical characteristics of adolescents reporting psychotic symptoms with adolescents who did not report such symptoms. Second, we used logistic regression analyses to examine the association between the outcome measure, experiencing psychotic symptoms, and the exposures: child physical and sexual abuse, exposure to domestic violence and bullying (victimisation or perpetration). We report univariate associations in terms of odds ratios, along with 95\% confidence intervals (95\% CI) and $P$-values, adjusted for gender and socio-economic status. Third, in order to control for the effect of comorbid psychiatric illness, we used a regression analysis stratified by the presence of psychiatric disorder. All analyses were carried out using STATA version 8.2 for Windows.

\section{Results}

Of the 211 children interviewed, $83(39 \%)$ received a DSM-IV ${ }^{17}$ Axis 1 diagnosis following $\mathrm{K}-\mathrm{SADS}$ interview. The most common disorders diagnosed were depressive disorders. No participants received a formal diagnosis of a psychotic illness. Fourteen participants $(6.6 \%)$ reported experiencing psychotic symptoms, primarily auditory and visual hallucinatory experiences. Adolescents who reported psychotic symptoms were significantly more likely to receive an Axis 1 psychiatric diagnosis than were adolescents who reported no such symptoms $(71.4 \%$ v. $37.1 \%$; $\chi^{2}=5.4$, d.f. $=1, P=0.02$ ). Specifically, the diagnoses of the 14 adolescents who reported psychotic symptoms included eight depressive disorders, two conduct disorders, two phobic disorders, two attention-deficit hyperactivity disorders, and one each of post-traumatic stress disorder, overanxious disorder, oppositional defiant disorder, tic disorder and separation anxiety disorder.

Adolescents who reported psychotic symptoms were more likely to be male $(71.4 \%$, psychotic symptoms group $v$. $45.2 \%$, comparison group; $\chi^{2}=3.3$, d.f. $\left.=1, P=0.07\right)$, and from high socio-economic groups (professional/managerial) $(64.3 \% \quad v$. $38.1 \%$; $\chi^{2}=3.5$, d.f. $\left.=1, P=0.06\right)$ than the adolescents who did not report any symptoms. There were no differences between the groups for family history of psychiatric illness $\left(\chi^{2}=0.12\right.$, d.f. $=1$, $P=0.73$ ).

Table 1 shows that adolescents reporting psychotic symptoms were six times more likely to have experienced child physical abuse than adolescents who did not report such symptoms and were ten times more likely to have witnessed domestic violence in their homes. These findings were statistically significant. Adolescents who reported psychotic symptoms were four-times more likely to have experienced child sexual abuse but, given the low absolute numbers of those who reported sexual abuse $(n=4)$, this difference was not statistically significant. Adolescents with psychotic symptoms were not significantly more likely to have been victims 


\begin{tabular}{|c|c|c|c|c|}
\hline \multirow[b]{2}{*}{ Trauma type } & \multicolumn{2}{|c|}{$\begin{array}{l}\text { (A) Adolescents with ( } n=14) v \text {. without }(n=197) \\
\text { psychotic symptoms }\end{array}$} & \multicolumn{2}{|c|}{$\begin{array}{l}\text { (B) Adolescents with psychiatric disorder with }(n=10) \\
\quad v \text {. without }(n=74) \text { psychotic symptoms }\end{array}$} \\
\hline & Odds ratio $(95 \% \mathrm{Cl})$ & $P$ & Odds ratio $(95 \% \mathrm{Cl})$ & $P$ \\
\hline Child physical abuse & $5.96(1.27-27.97)$ & 0.023 & $6.19(1.05-36.29)$ & 0.040 \\
\hline Child sexual abuse & $4.16(0.34-50.51)$ & 0.260 & $5.39(0.26-111.17)$ & 0.280 \\
\hline Exposure to domestic violence & $10.06(2.20-46.01)$ & 0.003 & $7.78(1.47-41.28)$ & 0.016 \\
\hline History of being bullied (victim) & $1.23(0.40-3.83)$ & 0.720 & $1.51(0.38-5.92)$ & 0.560 \\
\hline History of being a bully & $9.90(2.51-39.05)$ & 0.001 & $12.32(2.40-63.35)$ & 0.003 \\
\hline
\end{tabular}

of bullying but were significantly more likely to be bullies than adolescents who did not report such symptoms. Figure 1 shows the percentages of participants in each group who experienced the given traumatic events.

There was a high level of comorbidity between reported psychotic symptoms and other childhood psychiatric disorder in this sample. Therefore, we considered that the traumatic experiences identified could be associated with general psychopathology rather than showing any specificity to psychotic symptoms. To test this hypothesis, we carried out a further analysis confined to those adolescents who received psychiatric diagnoses following interview. The prevalence of childhood traumatic experiences among adolescents with a diagnosed psychiatric disorder who also displayed psychotic symptoms $(n=10)$ was compared with the prevalence among adolescents with diagnosed psychiatric disorder who did not display psychotic symptoms $(n=73)$. Table 1 shows that the association between psychotic symptoms and traumatic experiences remained statistically significant after controlling for psychiatric disorders.

\section{Discussion}

Our results show significant associations between psychotic symptoms in early adolescence and reports of child physical abuse, exposure to domestic violence and involvement in bullying. We did not find a significant association with child sexual abuse but the reported rate of sexual abuse in our sample as a whole was

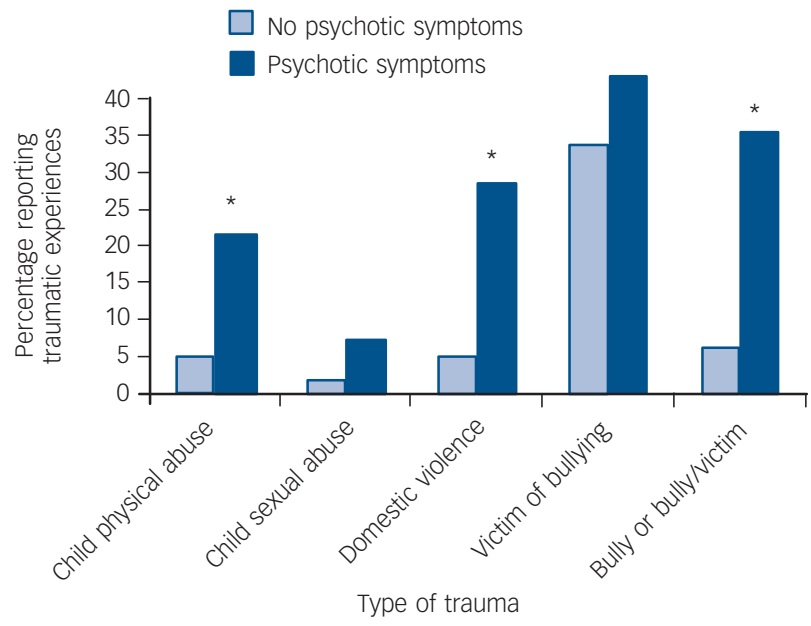

Fig. 1 Percentage of adolescents with and without psychotic symptoms reporting traumatic experiences.

${ }^{*} P<0.05$ low. There is an emerging literature on the association between adverse childhood experiences and positive psychotic symptoms, and this literature has focused mainly on child physical and sexual abuse. ${ }^{1,2}$ We have extended these findings to exposure to domestic violence and bullying, which have been rarely studied to date. ${ }^{4}$

\section{Traumatic experiences and mechanisms of risk for psychosis}

Theories proposed to explain how exposure to childhood abuse may increase the risk of psychotic symptoms have drawn on findings that severe stress at a young age appears to result in catecholamine deregulation and hypothalamic-pituitary-adrenal axis dysfunction. ${ }^{18}$ It has been shown that adult female victims of child physical and sexual abuse demonstrate persistent sensitisation of the pituitary-adrenal and autonomic stress response. ${ }^{19}$ This process may create a diathesis that renders the individuals' mental health vulnerable to stress and may place them at greater risk of future psychiatric illness, including psychosis. ${ }^{20,21}$

Although direct evidence is lacking, witnessing domestic (interparental) violence may well invoke similar stress-induced neurodevelopmental changes as those evidenced in victims of childhood physical and sexual abuse. It is known that multiple behavioural, emotional, social and psychological effects are associated with childhood exposure to domestic violence. ${ }^{22,23} \mathrm{~A}$ correlation between marital discord and child urinary dopamine levels has also been reported, ${ }^{24}$ which may indicate a process of dopamine sensitisation. ${ }^{25}$

Complementary theories suggest that the cognitive schema formed as a result of traumatic experiences increase risk of psychosis ${ }^{1}$. These are characterised by negative schematic models of the self and the world. Abusive experiences, for example, may teach the victim that people are dangerous, ultimately leading to paranoid ideation and the appraisal of normal events as threatening. ${ }^{26-28}$

\section{Bullying and psychosis}

In the context of our findings on child physical abuse and exposure to domestic violence, our findings regarding bullying were somewhat counterintuitive. We found that adolescents who reported psychotic symptoms were not significantly more likely to have been victims of bullying than the remainder of the sample. This finding is contradictory to a number of studies that reported a significant association between psychotic symptoms and being a victim of bullying..$^{29,30}$ There are a number of possible explanations for this discrepancy. First, there were high reported baseline rates of bullying victimisation in our whole sample (34\%), which is likely to have reduced our ability to find an effect of victimisation. We did not have sufficient information on the nature of the 
victimisation experiences to allow subdivision by severity of victimisation. It is worth noting that, of those identified as bullies in the psychotic symptom group, the majority $(80 \%)$ were also victims of bullying and would fit the definition of 'bully/victim' rather than the typical bully profile. In fact, children who fit the definition of bully/victim appear to be at particularly high risk of psychotic experiences, as $50 \%$ of the bully/victims in the study reported psychotic symptoms. Research is beginning to address the factors associated with bully/victim status. ${ }^{31,32}$ Compared with 'pure' victims, they tend to develop more pervasive and more severe psychological and behavioural outcomes, even after controlling for adjustment problems existing prior to the bully/ victim behaviours. ${ }^{32}$ Studies suggest that bully/victims come from homes where the parents are less involved and more likely to be hostile and rejecting. ${ }^{33}$

Physical abuse and exposure to domestic violence may be common risk factors for both the development of psychotic symptoms and the development of bullying behaviour. Children who have witnessed domestic violence or who have been victims of physical abuse may model their own behaviour on such acts of aggression. It could also be hypothesised that underlying genetic factors may influence antisocial and violent behaviour both in the parents and in the child. However, we found no significant relationship between being a bully and experiencing physical abuse and/or domestic violence. More research is needed to clarify what role bullying behaviour may play in the development of psychotic symptoms.

\section{Strengths and limitations}

This study has a number of strengths. First, it was populationbased and all participants were attending mainstream schools. The detailed clinical interviews provided a comprehensive evaluation of the prevalence of experiences of trauma. Second, parents were interviewed in every instance and this collateral information was particularly valuable regarding child abuse and exposure to domestic violence. Parents, when reporting traumas, were unaware that their offspring had reported psychotic symptoms. Furthermore, most studies on psychosis that have investigated child abuse have been conducted with adults. There is, therefore, a significant intervening time period between the abuse events and assessment of these events. In our study the assessment is much closer to the time of abuse events. All of these factors reduce the possibility of recall bias, which has been identified as a significant problem in the literature to date. ${ }^{2}$

One limitation is that the Challenging Times study was not designed specifically to identify psychotic symptoms. This may have resulted in less sensitivity in detecting weaker symptoms with an underestimation of symptom prevalence in this sample. Another important limitation is the cross-sectional nature of the study. Our ability to draw causal conclusions between traumatic experiences and psychotic symptoms is limited by a lack of clear temporal information. If psychotic symptoms preceded trauma in only a couple of the cases this would have serious implications since the numbers with psychotic symptoms is small. However, our data from parental interviews show that exposure to domestic violence, at least, occurred from a very young age, typically less than 5 years old.

The questions on sexual abuse in the interview instrument involve a somewhat narrow definition of abuse and the ambiguity of these questions may partially explain the low sexual abuse rates reported in this study. One study, based on self-report questionnaires, has suggested that sexual abuse (or, more specifically, 'unwanted sexual experiences') is, in fact, more common among adolescents who report having experienced psychotic symptoms. ${ }^{30}$
Although the present study was population based, it does not represent a true population cohort since one of the two groups was selected for being 'at risk' of psychiatric problems, based on screening measures. However, we have controlled for psychiatric disorders in our analysis. There was also a higher than expected refusal rate among the comparison group. Although the overall sample size was large, there were small numbers with the symptoms of interest and with the experiences of interest (particularly sexual abuse). For these reasons, replication of our findings is warranted.

\section{Early life traumatic events and psychosis}

Clearly not all individuals who experience severely traumatic events in childhood go on to develop a psychotic disorder. Disease causation is a dynamic process and should be considered in terms of a pathway over a life course rather than in terms of a certain set of risk factors at a point in time. ${ }^{34}$ Other 'environmental' risk factors for schizophrenia, such as obstetric complications, ${ }^{35}$ urbanicity $^{36}$ or cannabis use, ${ }^{37}$ may have cumulative or interactive effects on psychosis risk. None the less, this paper adds to the evidence that traumatic childhood events may be part of a cascade that leads to the development of psychotic symptoms and may ultimately contribute to the onset of psychotic illness.

Ian Kelleher, MSc, Department of Psychiatry, Royal College of Surgeons in Ireland Dublin, Ireland; Michelle Harley, MB, MRCPsych, Department of Psychiatry, Royal College of Surgeons in Ireland, and Child and Adolescent Mental Health Service, Mater Misericordiae University Hospital, Dublin, Ireland; Fionnuala Lynch, MB, MD, MRCPsych, MSc, Child and Adolescent Mental Health Service, Mater Misericordiae University Hospital, and University College Dublin, Ireland; Louise Arseneault, PhD, Medical Research Council Social, Genetic and Developmental Psychiatry Centre, Institute of Psychiatry, King's College London, UK; Carol Fitzpatrick, MD, FRCPI, FRCPsych, Child and Adolescent Mental Health Service, Mater Misericordiae University Hospital, The Children's University Hospital Temple St, and University College Dublin, Ireland; Mary Cannon, MB, PhD, MRCPsych, Department of Psychiatry, Royal College of Surgeons in Ireland and Beaumont Hospital, Dublin, Ireland.

Correspondence: Professor Mary Cannon, Department of Psychiatry, Royal College of Surgeons in Ireland, Education and Research Centre, Beaumont Hospital, Dublin 9, Ireland. Email: marycannon@rcsi.ie

First received 9 Jan 2008, final revision 4 Jul 2008, accepted 24 Jul 2008

\section{Acknowledgements}

This work was supported by a Clinician Scientist Award to M.C. (CSA/2004/1) from the Health Research Board (Ireland). LA is supported by a Career Scientist Award from the Department of Health (UK). We thank Ms Carla Mills and Ms Irene Daly for their roles in conducting clinical interviews for this study. The original Challenging Times study was funded by Friends of the Children's University Hospital (Dublin), the American Foundation for Suicide Prevention, HSE Northern Area and the Mater Misericordiae University Hospital.

\section{Appendix}

\section{K-SADS Psychosis section ${ }^{15}$ (reprinted with permission)}

\section{Hallucinations:}

Sometimes children, when they are alone, hear voices or see things, or smell things and they don't quite know where they come from. Has this ever happened to you? Tell me about it.

Has there ever been a time you heard voices when you were alone? What did you hear?

Have you ever heard someone call your name when there was no one around?

What kind of things did you hear?

Did you ever hear music which other people could not?

Has there ever been a time when you saw things that were not there?

What about shadows or other objects moving?

Did you ever see ghosts? When? 
Did this only happen at night while you were trying to sleep, or did it happen in the daytime too? What did you see?

Has there ever been a time when you had an unusual smell about yourself?

\section{Delusions}

Do you know what imagination is? Tell me.

Has there ever been a time your imagination played tricks on you? What kinds of tricks? Tell me more about them.

Did you have any ideas about things that you didn't tell anyone because you are afraid they might not understand? What were they?

Did you believe in things that other people didn't believe in? Like what?

\section{References}

1 Read J, van Os J, Morrison AP, Ross CA. Childhood trauma, psychosis and schizophrenia: a literature review with theoretical and clinical implications. Acta Psychiatr Scand 2005; 112: 330-50.

2 Morgan C, Fisher H. Environmental factors in schizophrenia: childhood trauma - a critical review. Schizophr Bull 2007; 33: 3-10.

3 Spataro J, Mullen PE, Burgess PM, Wells DL, Moss SA. Impact of child sexual abuse on mental health: prospective study in males and females. Br J Psychiatry 2004; 184: 416-21.

4 Bebbington $P E$, Bhugra $D$, Brugha $T$, singleton $N$, Farrell $M$, Jenkins R, Lewis G, Meltzer H. Psychosis, victimisation and childhood disadvantage: evidence from the second British National Survey of Psychiatric Morbidity. Br J Psychiatry 2004; 185: 220-6.

5 Janssen I, Krabbendam L, Bak M, Hanssen M, Vollebergh W, De Graff R, van Os J. Childhood abuse as a risk factor for psychotic experiences. Acta Psychiatrica Scand 2004; 109: 38-45.

6 Spauwen J, Krabbendam L, Lieb R, Wittchen $\mathrm{H}-\mathrm{U}$, van Os J. Impact of psychological trauma on the development of psychotic symptoms: relationship with psychosis proneness. Br J Psychiatry 2006; 188: 527-33.

7 Meyer I, Muenzenmaier K, Cancienne J, Struening E. Reliability and validity of a measure of physical and sexual abuse histories among women with serious mental illness. Child Abuse Negl 1996; 2: 213-9.

8 Read J, Agar K, Argyle N, Aderhold V. Sexual and physical assault during childhood and adulthood as predictors of hallucinations, delusions and thought disorder. Psychol Psychother Theory Res Pract 2003; 76: 1-22.

9 Poulton R, Caspi A, Moffitt TE, Cannon M, Murray R, Harrington HL. Children's self-reported psychotic symptoms and adult schizophreniform disorder: a 15-year longitudinal study. Arch Gen Psychiatry 2000; 57: 1053-8.

10 Lynch F, Mills M, Daly I, Fitzpatrick C. Challenging Times: a study to detect Irish adolescents at risk of psychiatric disorders and suicidal ideation. J Adolesc 2004; 27: 441-51.

11 Lynch F, Mills M, Daly I, Fitzpatrick C. Challenging Times: prevalence of psychiatric disorders and suicidal behaviours in Irish adolescents. J Adolesc 2006; 29: 555-73.

12 Goodman R. The strengths and difficulties questionnaire: a research note. $J$ Child Psychol Psychiatry 1987; 38: 582-6.

13 Goodman R, Ford T, Simmons H, Gatward R, Meltzer H. Using the Strengths and Difficulties Questionnaire (SDQ) to screen for child psychiatric disorders in a community sample. Br J Psychiatry 2000; 177: 534-9.

14 Kovacs M. The Children's Depression Inventory (CDI). Psychopharmacol Bull 1985; 21: 995-7.

15 Kaufman J, Birmaher B, Brent D, Rao U, Ryan N. The Schedule for Affective Disorders and Schizophrenia for School Aged Children: Present and Lifetime Version. University of Pittsburgh, Western Psychiatric Institute and Clinic, 1996.

16 Central Statistics Office Ireland. Census of population, 1996. Government of Ireland, 1996.
17 American Psychiatric Association. Diagnostic and Statistical Manual of Mental Disorders (4th edn) (DSM-IV). APA, 1994

18 Read J, Perry BD, Moskowitz A, Connolly J. The contribution of early traumatic events to schizophrenia in some patients: a traumagenic neurodevelopmental model. Psychiatry 2001; 64: 319-45.

19 Heim C, Newport D, Heit S, Graham YP, Wilcox M, Bonsall R, Miller AH, Nemeroff $\mathrm{CB}$. Pituitary-adrenal and autonomic responses to stress in women after sexual and physical abuse in childhood. JAMA 2000; 284: 592-7.

20 Walker E, DiFiori D. Schizophrenia: a neural diathesis-stress model. Psychol Rev 1997; 104: 667-85.

21 Cotter $D$, Pariante CM. Stress and the progression of the developmental hypothesis of schizophrenia. Br J Psychiatr 2002; 181: 363-5.

22 Jaffee S, Moffitt TE, Caspi A, Taylor A, Arseneault LA. Influence of adult domestic violence on children's internalising and externalising problems: an environmentally informative twin study. J Am Acad Child Adolesc Psychiatr 2002; 41: 1095-103.

23 Osofsky JD. The impact of violence on children. Future Child 1999; 9: 33-59.

24 Gottman JM, Katz LF. The effects of marital discord on young children's peer interaction and health. Dev Psychol 1989; 25: 373-81.

25 Cougnard A, Marcelis M, Myin GI, De Graaf R, Vollebergh W, Krabbendam L, Lieb R, Wittchen H-U, Henquet C, Spauwen J, Van Os J. Does normal developmental expression of psychosis combine with environmental risk to cause persistence of psychosis? A psychosis-persistence model. Psychol Med 2007; 37: 513-27.

26 Kuipers E, Garety P, Fowler D, Freeman D, Dunn G, Bebbington P. Cognitive, emotional, and social processes in psychosis: refining cognitive behavioural therapy for persistent positive symptoms. Schizophr Bull 2006; 32: s24-31.

27 Bentall RP, Corcoran R, Howard R, Blackwood N, Kinderman P. Persecutory delusions: a review and theoretical integration. Clin Psychol Rev 2001; 21: 1143-92.

28 Birchwood $M$, Meaden $A$, Trower $\mathrm{P}$, Gilbert $\mathrm{P}$, Plaistow J. The power and omnipotence of voices: subordination and entrapment by voices and significant others. Psychol Med 2000; 30: 337-44.

29 Lataster T, van Os J, Drukker M, Henquet C, Feron F, Gunther N, Myin-Germeys I. Childhood victimisation and developmental expression of non-clinical delusional and hallucinatory experiences. Soc Psychiatry Psychiatr Epidemiol 2006; 41: 423-8.

30 Campbell MLC, Morrison AP. The relationship between bullying, psychotic like experiences and appraisals in 14-16-year olds. Behav Res Ther 2007; 45: 1579-91.

31 Veenstra R, Lindenberg S, Oldehinkel AJ, Dewinter AF, Verhulst FC, Ormel J. Bullying and victimization in elementary schools: a comparison of bullies, victims, bully/victims, and uninvolved preadolescents. Dev Psychol 2005; 41: 672-82.

32 Arseneault L, Walsh E, Trzesniewski K, Newcombe R, Caspi A, Moffitt TE. Bullying victimization uniquely contributes to adjustment problems in young children: a nationally representative cohort study. Pediatrics 2006; 118: 130-8.

33 Bowers L, Smith PK, Binney V. Perceived family relationships of bullies, victims and bully/victims in middle childhood. J Soc Pers Relat 1994; 11: 215-32.

34 Cannon M, Clarke MC. Risk for schizophrenia - broadening the concepts, pushing back the boundaries. Schizophr Res 2005; 79: 5-13.

35 Clarke MC, Harley M, Cannon M. The role of obstetric events in schizophrenia. Schizophr Bull 2006; 32: 3-8.

36 Krabbendam L, van Os J. Schizophrenia and urbanicity: a major environmental influence - conditional on genetic risk. Schizophr Bull 2005, 31: 795-9.

37 Caspi A, Moffitt TE, Cannon M, McClay J, Murray R, Harrington H-L, Taylor A, Arseneault L, Williams B, Braithwaite A, Poulton R, Craig IW. Moderation of the effect of adolescent-onset cannabis use on adult psychosis by a functional polymorphism in the COMT gene: Iongitudinal evidence of a gene $\times$ environment interaction. Biol Psychiatr 2005; 57: 1117-27. 